\title{
Damien W. M. Arrigan (Ed): Electrochemical Strategies in Detection Science
}

\author{
Grégoire Herzog ${ }^{1}$
}

(C) Springer-Verlag GmbH Germany 2017

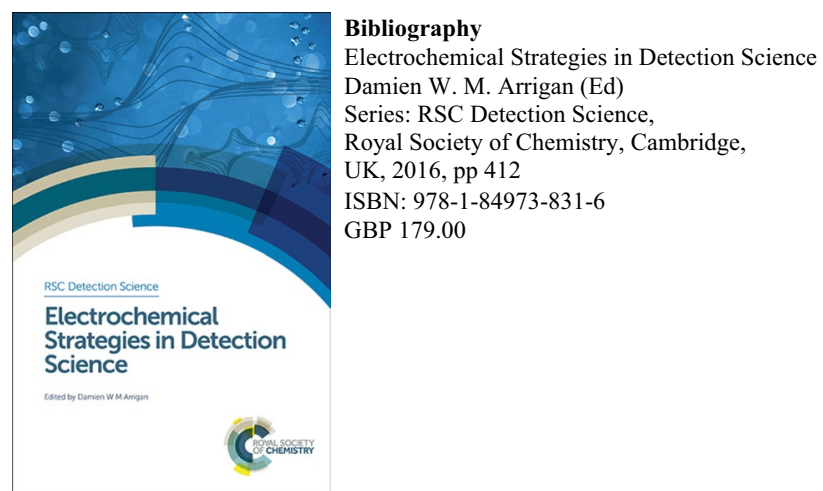

It is a fantastic time to be an electrochemist! Many of the challenges that scientists have to tackle in today's society involve, in one way or another, charge transfer across an interface. These challenges cover the fields of energy, water deionization, electronic manufacturing, material synthesis, or, as in the topic of this book, sensors. The need for simpler, faster, better, and cheaper detection methods is valid for many research disciplines, as well as for manufacturing industries and human activities such as health and environmental monitoring.

Electrochemical Strategies in Detection Science is the sixth volume of a Royal Society of Chemistry series dedicated to the advances of the field of Detection Science. This particular item is focused on the most recent updates in electrochemical detection methods. The volume gathers ten chapters, which can be divided into three groups. Three of these chapters focus on the detection of specific targets such as heavy metals (Chapter 1), nanoparticles (Chapter 5),

Grégoire Herzog

gregoire.herzog@univ-lorraine.fr

1 CNRS, Université de Lorraine, Villers-les-Nancy, France and ions (Chapter 9). Four chapters are oriented towards the fabrication and the applications of electroanalytical systems: microelectrode arrays for biomedical applications (Chapter 2); microchip electrophoresis (Chapter 3); scanning electrochemical microscopy for life science applications (Chapter 4); and nanoelectrodes (Chapter 6). Finally, the three remaining chapters review the state of the art of materials for electrochemical detection: carbon electrodes (Chapter 7); dispersible electrodes (Chapter 8); and ionic liquid-based electrodes (Chapter 10). In approximately 40 pages, each of the chapters presents the basic concepts necessary to understand the fundamentals of the topic before reviewing the most exciting and recent achievements published in the literature. This selection of chapters is a mix between well-established electroanalytical detection methods-e.g., stripping analysis of heavy metals (Chapter 1) and electroanalysis at carbon nanomaterials such as carbon nanotubes and graphene (Chapter 7) - and rather novel research themes such as the electrochemical detection of nanoparticles (Chapter 6) and the concept of dispersible electrodes (Chapter 8). Most of the chapters of Electrochemical Strategies in Detection Science are of an excellent standard, written by leading researchers in the field.

This volume offers a comprehensive snapshot of the current state of the art of electrochemical detection methods. Its tutorial tone will enlighten both specialist and non-specialist readerships eager to discover the field and will contribute to guaranteeing a long shelf life. The variety of electrochemical strategies put forward highlights the vital role of electrochemistry in analytical sciences today. 